\title{
Functionalized Iron Oxide - Metal Hybrid Nanoparticles for Protein Extraction from Complex Fluids
}

Akanksha Gurtu, Nahida Akhtar, Meenakshi Verma, Kultar Singh, Georgette MoyleHermann, Mandeep Singh Bakshi

Fig S1

(a)

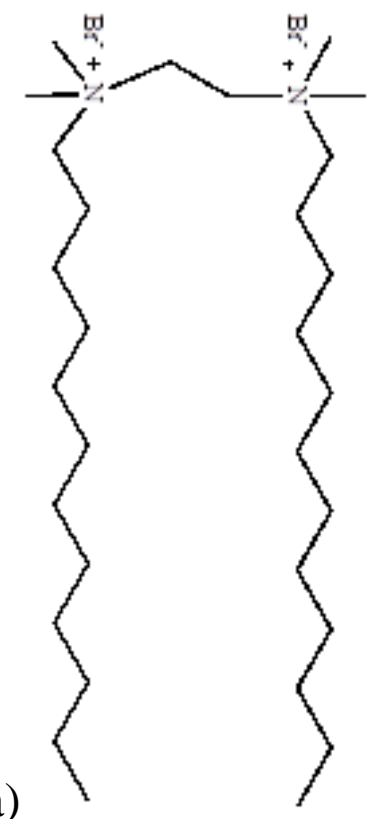

(b)

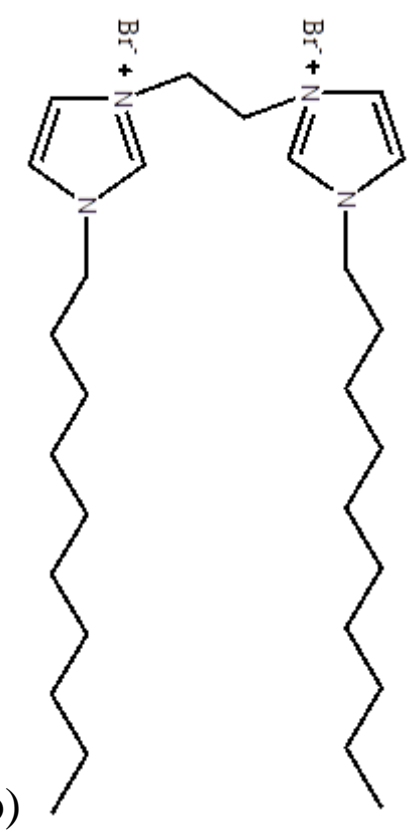

Fig S1. General molecular structures of 12-2-12 or 16-2-16 (a) and C14S4 (b). 
Fig S2

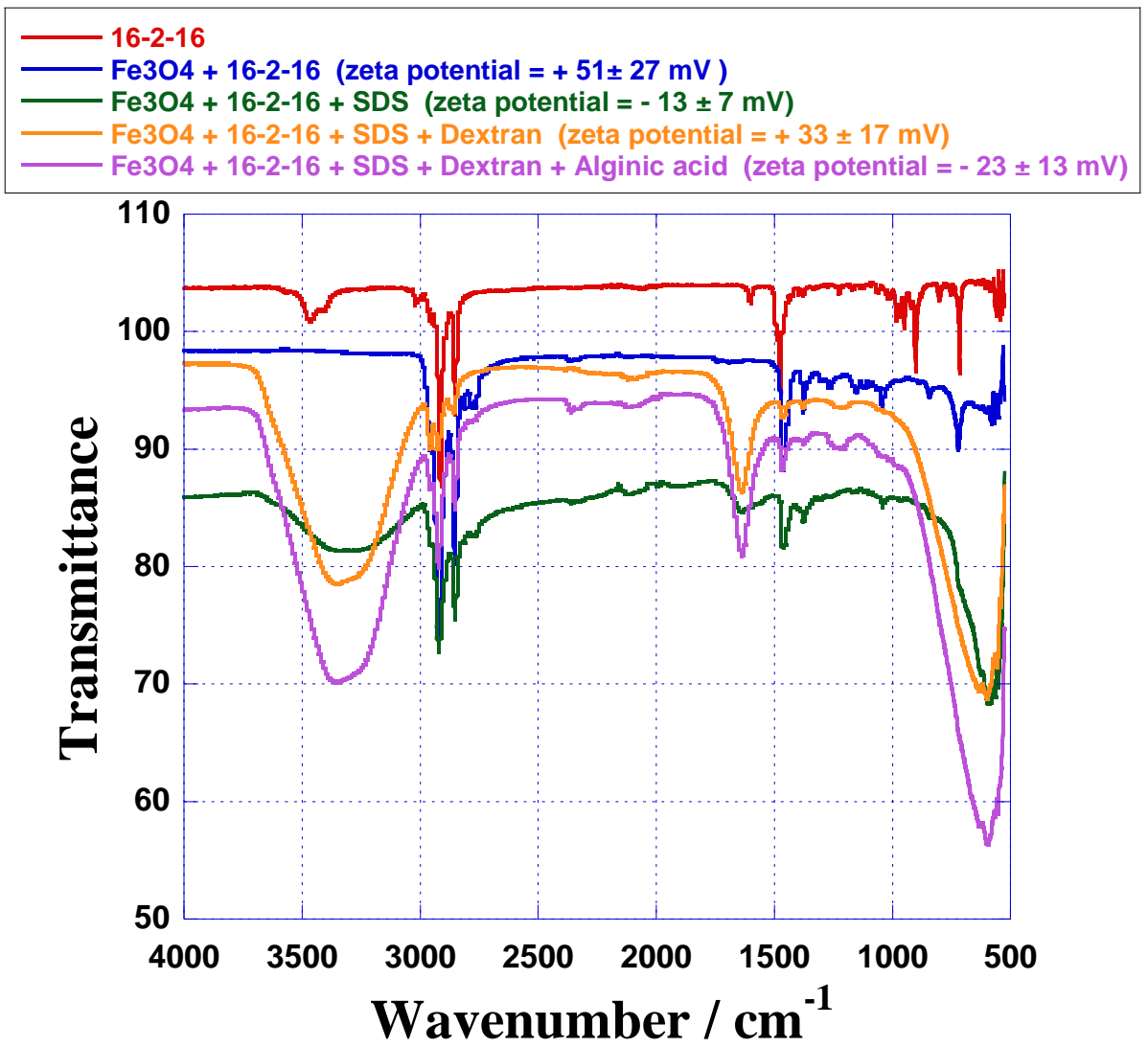

Fig S2. FTIR spectra of purified magnetic NPs after treating them with different coating agents. Zeta potentials of the respective samples are shown in the parentheses.

The FTIR spectra of Gemini surfactant 16-2-16 (shown in the above figure, 16-216) show the following absorption bands near the frequencies: $714.80-729.72 \mathrm{~cm}^{-1}$ $\left(\mathrm{CH}_{2}\right.$ rocking), 1472.27-1472.61 $\mathrm{cm}^{-1}\left(\mathrm{CH}_{2}\right.$ deformation), 2851 and $2921 \mathrm{~cm}^{-1}$ $\left(\mathrm{CH}_{\mathrm{st}}\right)$ and $3463 \mathrm{~cm}^{-1}\left(\mathrm{CN}^{+}\right)$. These FTIR spectra confirm the presence of tetraalkylammonium head group and long hydrocarbon chain functional groups for twin tail 16-2-16 Gemini surfactant.

The 16-2-16 is amphiphilic with two hydrophobic tails connected to hydrophilic dimeric head groups $\left(\mathrm{R}_{4} \mathrm{~N}^{+}\right)$. This surfactant binds to $\mathrm{Fe}_{3} \mathrm{O}_{4}$ NPs through 
hydrophilic head groups (see $\mathbf{F e}_{3} \mathbf{O}_{4}+\mathbf{1 6 - 2 - 1 6}$ ). That causes the absorption bands due to hydrophobic tails i.e. 714.80-729.72 $\mathrm{cm}^{-1}\left(\mathrm{CH}_{2}\right.$ rocking), 1472.27-1472.61 $\mathrm{cm}^{-1}\left(\mathrm{CH}_{2}\right.$ deformation), 2851 and $2921 \mathrm{~cm}^{-1}\left(\mathrm{CH}_{\mathrm{st}}\right)$ remain unaffected. However, the binding of hydrophilic head group causes the disappearance of peak at 3463 $\mathrm{cm}^{-1}$ indicating a strong binding of 16-2-16 on $\mathrm{Fe}_{3} \mathrm{O}_{4}$ NPs surface.

The second coating on the NPs was carried out with SDS (see $\mathbf{F e}_{3} \mathbf{O}_{\mathbf{4}}+\mathbf{1 6 - 2 - 1 6}+$ SDS). This surfactant has one hydrophobic tail and hydrophilic head. The second layer gets coated through hydrophobic tail as the peak due to $\mathrm{CH}_{2}$ rocking at 722 $\mathrm{cm}^{-1}$ disappears but the peaks due to $\mathrm{CH}_{\text {str }}$ remain unaffected. Further, a broad peak in the fingerprint region suggests that after second coating with SDS, the interactions between $\mathrm{M}-\mathrm{N}$ gets strengthened.

The third coating on NPs was carried out with DEAE Dextran (see $\mathbf{F e}_{3} \mathbf{O}_{4}+\mathbf{1 6 - 2}$ $16+$ SDS + Dextran). As Dextran is a polycationic compound, so its positively charged group interact with sulphate groups of SDS and this coating further intensify and broaden the peak due to $\mathrm{M}-\mathrm{N}$ in fingerprint region suggesting the more stability of the coated NPs. This coating also increases the vibrational frequency of $\mathrm{CH}_{\text {str }}$ which now appear at 2911 and $2955 \mathrm{~cm}^{-1}$. An additional sharp peak at $1633 \mathrm{~cm}^{-1}$ is due to alkoxy C-O group of dextran and a broad band near $3400 \mathrm{~cm}^{-1}$ appears due to $\mathrm{O}-\mathrm{H}$ functional groups of dextran.

The fourth coating on NPs (see $\mathrm{Fe}_{3} \mathrm{O}_{4}+\mathbf{1 6 - 2 - 1 6}+\mathrm{SDS}+$ Dextran + Alginic acid) was carried out with a hydrophilic colloidal polysaccharide alginic acid. The encapsulation of NPs with alginic acid, further intensify and broadened of the bond at $3400 \mathrm{~cm}^{-1}$ due to $\mathrm{O}-\mathrm{H}$ group. This encapsulation further increases the stability of NPs as confirmed by further broad and intense peak in fingerprint region due of M$\mathrm{N}$ bond. 
Fig S3

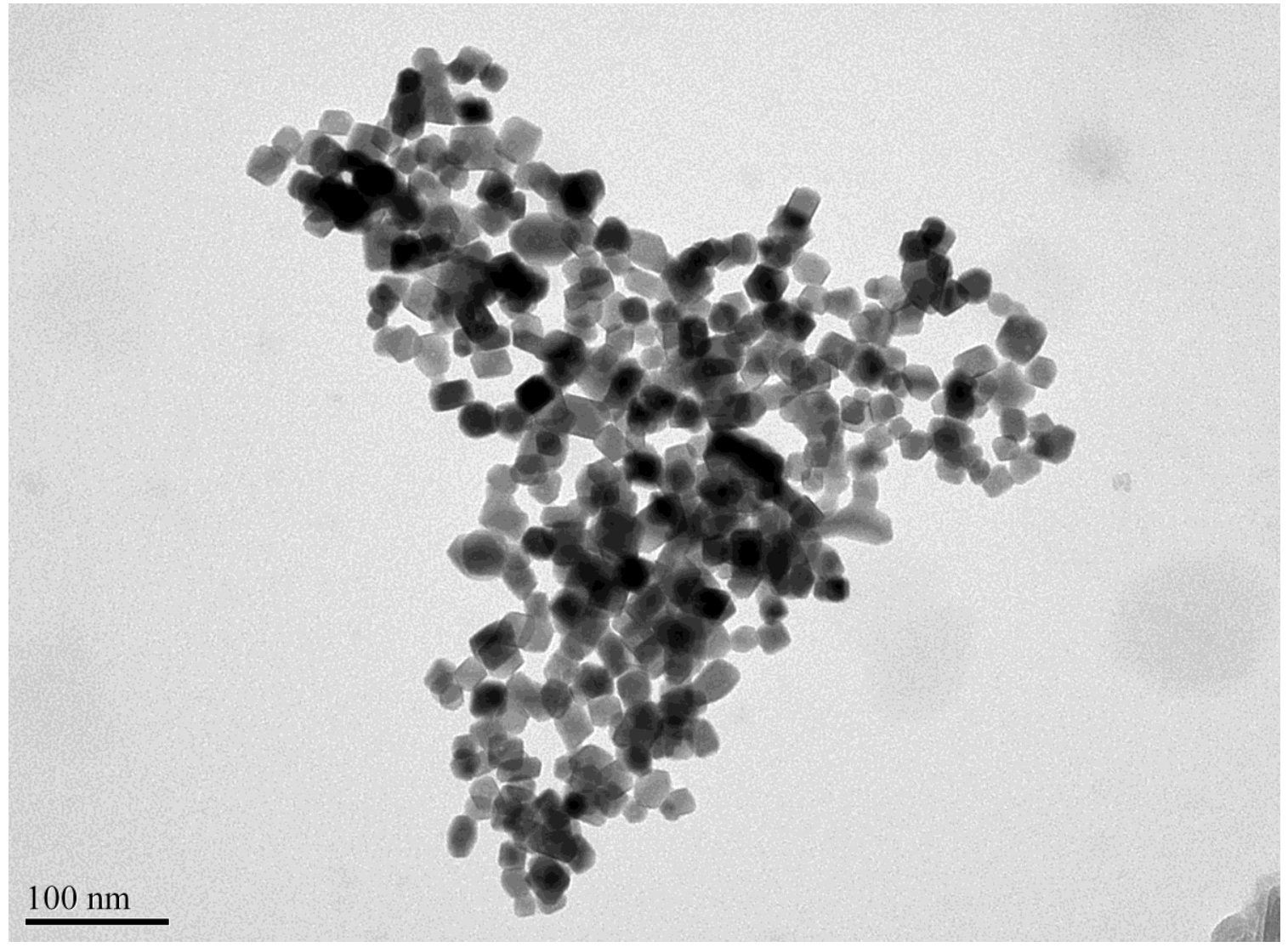

Fig S3. TEM micrograph of iron oxide NPs synthesised in the presence of 12-2-12. Please see experimental for detailed synthesis. 
Fig S4

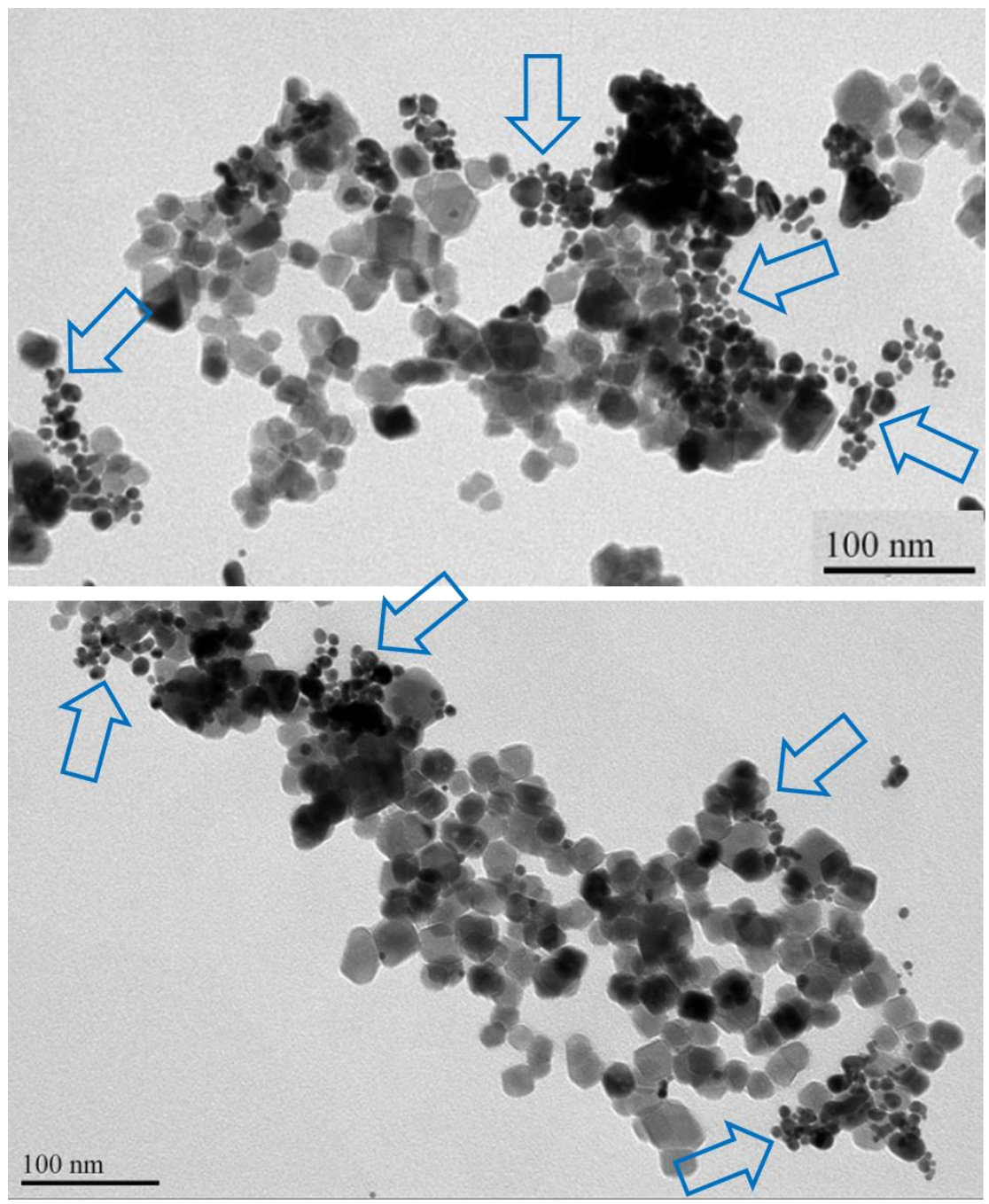

Fig S4. High resolution TEM micrograph of iron oxide - Au hybrid NPs. Small sized dark contrast Au NPs can easily differentiated from the large sized light contrast iron oxide NPs. Small Au NPs have been indicated by block arrows. Note that all Au NPs are either adsorb on the iron oxide NPs or they exist along with them. This association is mainly driven by the presence of coating ligands on the iron oxide NPs. 